\title{
Properties and natural occurrence of maternal-effect selfish genes ('Medea' factors) in the Red Flour Beetle, Tribolium castaneum
}

\author{
RICHARD W. BEEMAN* $\uparrow \&$ KENLEE S. FRIESEN $\dagger+$ \\ $\dagger$ Grain Marketing and Production Research Center, USDA, ARS, 1515 College Avenue, Manhattan, KS 66502, \\ U.S.A. and ¥Division of Biology, Kansas State University, Manhattan, KS 66506, U.S.A.
}

\begin{abstract}
Maternally acting selfish genes, termed 'Medea' factors, were found to be widespread in wild populations of Tribolium castaneum collected in Europe, North and South America, Africa and south-east Asia, but were rare or absent in populations from Australia and the Indian subcontinent. We detected at least four distinct genetic loci in at least two different linkage groups that exhibit the Medea pattern of differential mortality of genotypes within maternal families. Although each $M$ factor tested had similar properties of maternal lethality to larvae and zygotic self-rescue, $M$ factors representing distinct loci did not show cross-rescue. Alleles at two of these loci, $M^{1}$ and $M^{4}$, were by far the most prevalent, $M^{4}$ being the predominant type. $M^{2}$ and $M^{3}$ were each found only once, in Pakistan and Japan, respectively. Although $M^{1}$ could be genetically segregated from $M^{4}$ and maintained as a purified stock, the $M^{1}$ factor invariably co-occurred with $M^{4}$ in field populations, whereas $M^{4}$ usually occurred in the absence of other Medea factors. The dominant maternal lethal action of $M^{1}$ could be selectively inactivated (reverted) by gene-knockout gamma irradiation with retention of zygotic rescue activity.
\end{abstract}

Keywords: maternal effect, Medea factor, selfish gene, Tribolium.

\section{Introduction}

In 1992 we reported the discovery, in wild populations of Tribolium, of a class of selfish genes previously unknown in the animal kingdom (Beeman et al., 1992). These genes showed the unusual property of maternal lethality to hatchlings, combined with zygotic selfrescue. They were termed 'Medea' $(=M)$ factors, an acronym for Maternal Effect Dominant Embryonic Arrest. This mechanism ensures that progeny of a carrier mother survive only if they inherit a copy of the gene from either parent. Such a genetic element is predicted to spread in a population, even in the absence of any selective advantage conferred upon the host (Wade \& Beeman, 1994). Subsequently, a genetic disease in mice was shown to be associated with a selfish gene directly analogous to Medea (Hurst, 1993; Peters \& Barker, 1993), and Medea-like maternal and zygotic effects were observed for a region of mouse chromosome 1 bearing a high-copy long-range repeat cluster (Weic-

*Correspondence. E-mail: beeman@usgmrl.ksu.edu

(C) 1999 The Genetical Society of Great Britain. henhan et al., 1996). In the current work we (i) demonstrate the existence of at least 4 distinct Medea loci in wild populations of $T$. castaneum; (ii) confirm the absence of cross-rescue between nonallelic $M$ factors; (iii) show that maternal lethal activity of a Medea factor can be selectively reverted with retention of rescue activity; and (iv) examine on a global scale the distribution of the two commonly occurring $M$ factors in wild populations.

\section{Materials and methods}

\section{Description of strains}

Five standard laboratory strains, GA-1, $M^{1}, M^{4} a u, M^{1}$ $M^{4}$ and $3 P 1 / a u^{14}$, were used to screen field strains for the presence of Medea factors. GA-1 is a standard laboratory strain collected in a farmer's corn bin in Georgia (Haliscak \& Beeman, 1983) and is apparently devoid of Medea alleles. $M^{1}$ is homozygous for the $M^{1}$ factor (third linkage group) derived from the SP strain from Singapore (Beeman et al., 1992). $M^{4}$ au is 
homozygous for the visible recessive marker aureate $(a u)$, as well as for the incidental markers, Abdominalmissing abdominal sternites $\left(A^{\text {mas }}\right)$ and pearl $(p)$, and during the course of this work was found to be also homozygous for $M^{4}$, although it lacks $M^{1} \cdot M^{1} M^{4}$ is homozygous for the visible recessive markers microcephalic ( $m c$ ) and jet (j) (Sokoloff, 1962), both incidental to the present work, and during the course of this work was found to be also homozygous for both $M^{1}$ and $M^{4} .3 P 1$ and $3 P 2$ au are 3rd linkage group (LG) balancers (Mocelin \& Stuart, 1996) that carry the dominant visible marker, Blunt abdominal and metathoracic projections (Bamp) (Beeman, 1986; Beeman \& Stuart, 1990). Both $3 P 1$ and $3 P 2$ au eliminate crossing-over in a region that includes the Bamp, $a u$ and $M^{1}$ loci on LG3. $a u^{14}$ is a lethal aureate allele, and was used to maintain both $3 P 1$ and $3 P 2 a u$ as balanced lethal stocks. These balancer stocks are apparently devoid of $M^{1}$ alleles. Most field strains used in this study were collected from farms, grain storage facilities, mills, warehouses and food markets in North and South America, Europe, Africa, the Indian subcontinent, south-east Asia and Australia between 1985 and 1995, and have been maintained in the laboratory on whole wheat flour fortified with $5 \%$ brewers' yeast. Additional information is available at the Tribolium web site (http://bru.usgmrl.ksu.edu/beeman/tribolium.html).

\section{Detection and differentiation of Medea loci}

$M$ factors were initially detected in a subset of all field strains by crossing females from each strain with GA-1 males, then testcrossing $F_{1}$ females with GA-1 males in single pairs. In the presence of Medea, offspring of such $\mathrm{F}_{1}$ families will segregate $50 \%$ Medea heterozygotes (viable) and 50\% non-Medea homozygotes (inviable). Thus, the presence of an $M$ factor in the original field strain was indicated by the death of $\approx 50 \%$ of the hatchlings from the testcross. Dead hatchlings were never found in control crosses or in crosses involving non-Medea segregants. $M$ factors diagnosed in this way were then tested for genetic linkage and for cross-rescue. All $M$ factors were initially tested for linkage to $M^{l}$ on the third linkage group (LG) using the closely linked visible marker aureate (Beeman et al., 1992). Additional genetic mapping was carried out using backcrosses of multiple heterozygotes to the multiple homozygous recessive as described (Beeman et al., 1992). Apparent linkage of a new Medea with a recessive marker in trans was confirmed by separate linkage tests in cis. After initial detection and mapping, each apparent Medea factor was confirmed by demonstrating that hatchling kill was strictly maternal, and that it occurred only in non-Medea progeny. This was accomplished by testing
Medea-bearing males for progeny kill, and by testing surviving progeny of segregating Medea females for the presence of $M$ factors. Cross-rescue was tested by assessing the zygotic rescue activity of a paternally derived $M$ factor in an embryo under the lethal maternal influence of a different $M$ factor.

\section{Reversion of $\mathrm{M}^{1}$ lethality}

To confirm that the lethal and rescue activities of $M$ factors are controlled by separate (but tightly linked) genetic elements, we tested whether maternal lethal activity of $M^{1}$ could be selectively deleted by knockout mutation with retention of intact zygotic rescue activity. Screens for loss of maternal lethal activity were conducted after gamma irradiation of spermatozoa using a $\mathrm{Co}^{60}$ source. One-hundred homozygous $M^{1}$ au males 12 weeks of age were irradiated at a dose of $4 \mathrm{kR}$, then immediately crossed en masse to $2003 P 2 \mathrm{au} / \mathrm{au}$ virgin females. After 3 days the males were discarded and the females were allowed to oviposit for 1 month. $F_{1}$ virgin females heterozygous for $3 P 2 a u$ and for the treated $M^{1}$ au chromosome were testcrossed to 3P1/au males in single pairs. Revertants of maternal lethality were recognized by the presence of phenotypically Bamp, au beetles (presumably $3 P 2$ au/au) among the progeny. Because $3 P 2$ au/au progeny did not inherit $M^{I}$, they would not express rescue activity, and thus would normally be killed by maternal $M^{1}$. Putative revertants were tested to rule out false positives derived by recombination between $M^{1}$ and the balancer chromosome. Testcross progeny that were phenotypically Bamp, non-au (presumably $M^{l R} a u / 3 P 1$, where $\mathrm{R}=$ revertant) were used to establish balanced lethal stocks, or to determine whether the revertant was homozygous viable.

\section{Geographical distribution of Medea factors}

To survey wild populations more extensively for the presence of $M$ factors, separate screenings were conducted for each of three Medea categories, namely $M^{I}$, $M^{4}$, and 'all others'. Initially, two individuals were tested for each Medea type in each of 123 strains (total $=738$ beetles tested). These strains represented 25 source countries in Europe, the Middle East, North and South America, Africa, south-east Asia, Australia and the Indian subcontinent. For most strains, followup tests were carried out using 2-5 additional beetles for each Medea category (total $\approx 1400$ beetles tested). Diagnostic tests were as follows.

(i) To detect $M^{1}$, field strain males were crossed in single pairs with standard $M^{1} / 3 P 1$ virgin females and the adult progeny were scored for the $3 P 1$ (=Bamp) 
phenotype. If the field strain male was either heterozygous or homozygous for $M^{1}$, the maternally derived $3 P 1$ chromosome would be rescued from maternal lethality by a paternal $M^{1}$ chromosome. Thus, the presence or absence of Bamp progeny indicated the presence or absence, respectively, of a paternal $M^{1}$ allele. $M^{1}$ was confirmed in females from a subset of positive field strains by demonstrating the presence of $M^{1}$-linked maternal lethal activity. Test females from field strains were crossed to standard $M^{4}$ au males, and $\mathrm{F}_{1}$ females (potentially heterozygous for $M^{1}$ in trans with $a u$ ) were backcrossed to $M^{4}$ au males. The presence of an $M^{1}$ allele in trans with $a u$ in the $\mathrm{F}_{1}$ female would result in the death of almost all homozygous au progeny, because $a u$ is closely linked to $M^{l}$ (Beeman et al., 1992). Note that $M^{4}$-derived maternal lethality is completely suppressed in this backcross, as all progeny inherited an $M^{4}$ chromosome from their father, resulting in zygotic rescue.

(ii) $M^{4}$ alleles were detected by testing the ability of field strain males to rescue the maternal lethality associated with standard $M^{4} /+$ virgin females. The latter were generated by crossing GA-1 males with $M^{4}$ $a u$ virgin females, then collecting virgin $F_{1}$ females. Field strain males that lacked $M^{4}$ alleles failed to rescue the maternal lethality associated with $M^{4}$. Crosses involving such males were expected to result in $\approx 50 \%$ survival of progeny. Crosses involving males heterozygous for $M^{4}$ should show $\approx 75 \%$ progeny survival, whereas those employing homozygous $M^{4}$ males should give rescue of all progeny.

(iii) All other $M$ alleles were detected by testing field strains for the presence of maternal lethal activity that was not rescuable by standard alleles of either $M^{1}$ or $M^{4}$. $\mathrm{F}_{1}$ females derived from either of the testcrosses ( $\mathrm{i}$ and ii) described above were in turn crossed in single pairs with standard $M^{1} M^{4}$ males. Mortality of $50 \%$ of the hatchlings indicated the presence in the $F_{1}$ female of a field-strain-derived Medea factor other than $M^{1}$ or $M^{4}$, because the presence in all zygotes of paternally derived $M^{1}$ and $M^{4}$ factors would preclude any mortality associated with those elements. All standard strains either lack $M$ factors (other than $M^{l}$ or $M^{4}$ ) or are fixed for the same set of these 'other' $M$ factors, because all hatchling mortality associated with hybrids between standard strains used here is fully accounted for by the effects of either $M^{1}$ or $M^{4}$.

\section{Results}

\section{Medea alleles occur at four loci}

The $M^{1}$ Medea factor has been previously described and mapped to the far 'right' end of the 3rd linkage group, within one map unit of the au locus (Beeman et al., 1992). The $M^{l}$ allele examined in that work was derived from a strain collected in Singapore. New Medea factors characterized in the present work were first tested for linkage to $a u$ to determine whether they were potentially allelic with $M^{1}$. Preliminary tests revealed that several additional strains from south-east Asia carried $M$ factors that were closely linked to $a u$. One of these $\left(M^{2}\right)$, collected in Peshawar, Pakistan, was unique in that it did not show cross-rescue with $M^{1}$ (Table 1). When females doubly heterozygous for $M^{1}$ and $M^{2}$ in trans were testcrossed to males carrying various combinations of $M$ alleles, it became apparent that $M^{1}$ and $M^{2}$ were closely linked to each other and to $a u$, but that neither $M$ factor rescued the lethality of the other. A third $M$ factor, designated $M^{3}$ was also unique, being found in a single strain from Kukisaki, Ibaraki prefecture, Japan. This factor was mapped to the 8th linkage group, $\approx 11$ recombination units from antennapedia, away from squint (Table 2). The fourth Medea gene recognized $\left(M^{4}\right)$ was found in numerous laboratory and field strains from many sources. We have not yet succeeded in mapping $M^{4}$, but it assorts independently of the other three $M$ loci (data not shown). As expected, $M^{4}$ shows normal Mendelian segregation in heterozygous males and the unique 'selfish' mechanism (maternal lethal with zygotic self-rescue activity) that is the hallmark of this class of genetic elements (Table 3 and Thomson \& Beeman, 1997). In addition to the evidence presented above for the absence of cross-rescue between $M^{1}$ and $M^{2}$, we also have extensive evidence

Table 1 The closely linked Medea factors $M^{1}$ and $M^{2}$ do not cross-rescue

\begin{tabular}{|c|c|c|c|c|c|c|c|c|}
\hline \multirow{2}{*}{$\begin{array}{l}\text { Genotype of } \\
\text { male parent } \\
\text { Progeny } \\
\text { phenotype }\end{array}$} & \multicolumn{2}{|c|}{$M^{2} a u+$} & \multicolumn{2}{|c|}{$+a u M^{l}$} & \multicolumn{2}{|c|}{$+a u+$} & \multicolumn{2}{|c|}{$M^{2}$ au $M$} \\
\hline & $\mathrm{au}$ & + & $\mathrm{au}$ & + & $\mathrm{au}$ & + & $\mathrm{au}$ & + \\
\hline $\begin{array}{l}\text { Number of } \\
\text { progeny }\end{array}$ & 255 & 20 & 3 & 400 & 1 & 0 & 78 & 75 \\
\hline $\begin{array}{l}\% \text { survival } \\
\text { of progeny }\end{array}$ & \multicolumn{2}{|c|}{47} & \multicolumn{2}{|r|}{41} & \multicolumn{2}{|r|}{1} & \multicolumn{2}{|c|}{87} \\
\hline
\end{tabular}

Males (row 1) were taken from standard, homozygous stocks, and were crossed to $M^{2}++/+a u M^{l}$ females. These females were fullsibs, generated from a single-pair $M^{2}++/++a u$ female $\times a u M^{1}$ male. Data for each cross are pooled values from 4 to 5 single pairs. For crosses involving $M^{2} a u+$ or $+a u M^{1}$ males (columns 1-2) we allowed the single pairs to oviposit for 8 weeks prior to determination of progeny phenotypes. Percentage survival data are derived from retests of the same two sets of single pairs, based on number of eggs laid in a 3-day period. For crosses using $+a u+$ or $M^{2}$ au $M^{1}$ males (columns 3-4), a single, 3-day oviposition period was used both for assessment of progeny phenotypes and for measurement of percentage survival. 
Table 2 Assignment of $M^{3}$ to linkage group 8, and three-point mapping of $M^{3}$

\begin{tabular}{lrrrrrr}
\hline & \multicolumn{5}{c}{ Number of testcross progeny of given phenotype } & \\
\cline { 2 - 6 } Testcross $\dagger(\mathrm{f} \times \mathrm{m})$ & $\mathrm{a}$ & + & $\mathrm{s}$ & $\mathrm{a}, \mathrm{s}$ & Total & \% recomb $(M-a)$ \\
\hline$M+\mid+a \times+\mathrm{a} /+\mathrm{a}$ & 37 & 210 & - & - & 247 & 15 \\
$M a /++\times+a /+a$ & 251 & 32 & - & - & 283 & 11 \\
$+a /+a \times M+/+a$ & 56 & 70 & - & - & 126 & 44 \\
$M++\mid+a s \times+a s /+a s$ & 0 & 254 & 15 & 32 & 301 & 11 \\
\hline
\end{tabular}

Data for 1-3 single pairs were pooled for each testcross. Markers for linkage groups 1-5 and 7 were also tested, but none showed linkage to $M^{3}$ (data not shown).

$\dagger$ For each testcross, female genotype is given first.

Abbreviations: a, antennapedia; s, squint; $M$, Medea $^{3}$.

Table 3 Segregation of Medea factor $M^{4}$

\begin{tabular}{llc}
\hline & $\begin{array}{c}\text { No. of female progeny of } \\
\text { indicated genotype } \ddagger\end{array}$ \\
\cline { 2 - 3 } Segregation cross $\dagger$ & $M^{4} /+$ & $+/+$ \\
\hline$+/+\times M^{4} /+$ & 22 & 0 \\
$M^{4} /+\times+/+$ & 32 & 13 \\
$\dagger M^{4}$ heterozygotes used for reciprocal, single-pair testcrosses were \\
full-sibs, generated from a single-pair cross of GA-1 female $\times M^{4}$ \\
au male. For each segregation cross, female genotype is given first. \\
$\begin{array}{l}\ddagger \text { Progeny genotypes were determined by single-pair testcrosses to } \\
\text { standard GA-1 }(=+/+) \text { males, followed by measurement of }\end{array}$ \\
percentage survival. A $\approx 50 \%$ mortality rate at the hatching stage \\
indicated the $M^{4} /+$ maternal genotype.
\end{tabular}

that $M^{1}$ and $M^{4}$ do not show cross-rescue (data not shown). However, cross-rescue among $M^{2}, M^{3}$ and $M^{4}$ has not been tested.

\section{Selective reversion of $\mathrm{M}^{1}$ lethality}

We screened $\approx 1000$ irradiated $M^{1}$ au chromosomes for loss of maternal lethal activity and detected three apparent revertants. Two of these proved to be false positives, possibly resulting from recombination be- tween $M^{l}$ and the balancer chromosome. One true revertant $\left(=M^{l R}\right)$ was confirmed. A female $M^{1 R} a u / 3 P 2$ $a u$ testcrossed to a male $3 P 1 / a u$ produced phenotypically Bamp, au progeny (testcross 4 , Table 4 ). The latter must have been genotypically $3 P 2 a u / a u$, and would have been killed if the maternal $M^{1 R} a u$ chromosome had intact lethal activity (see control testcross 3, Table 4). Testcrosses 1 and 2 in Table 4 clearly show that a paternally derived $M^{1 R}$ au chromosome, lacking its own maternal lethal activity, could still rescue progeny from the maternal lethal effect of an intact $M^{1}$ chromosome. The revertant chromosome was homozygous lethal and was maintained for several generations as a true-breeding stock over the 3P1 balancer. However, it was associated with severely reduced fertility and eventually died out in spite of careful husbandry.

\section{World distribution of Medea types}

We tested 113 strains representing 23 countries for each of three Medea 'types': $M^{1}, M^{4}$ and $M^{X}$ (=all others). These 113 include only unique strains whose geographical origin is well-documented. The results, summarized in Table 5, reveal that $M^{1}$ and $M^{4}$ are the two predominant types worldwide, $M^{4}$ being by far the

Table 4 Selective reversion of $M^{l}$ lethality

\begin{tabular}{lrrrrr}
\hline & \multicolumn{5}{c}{ No. of progeny of given phenotype $\dagger$} \\
\cline { 2 - 6 } Testcross (female $\times$ male) + & B & + & au & B, au & Total \\
\hline $1 . M^{I} / a u \times M^{I R} a u / 3 P 1$ & 13 & 10 & $\mathbf{6}$ & 0 & 29 \\
$2 . M^{I} / 3 P 2 a u \times M^{I R} a u / 3 P 1$ & 22 & 5 & 0 & $\mathbf{9}$ & 36 \\
$3 . M^{1} / 3 P 2 a u \times a u / a u$ & $\mathbf{0}$ & 37 & 0 & $\mathbf{0}$ & 37 \\
$4 . M^{I R} a u / 3 P 2 a u \times 3 P 1 / a u$ & 4 & 0 & 15 & $\mathbf{4}$ & 23 \\
\hline
\end{tabular}

$\dagger$ Diagnostic phenotypes are in boldface type.

$\$$ All testcrosses are single pairs. Crosses $1-2$ test whether the $M^{I R}$ au revertant chromosome has intact rescue activity. Testcross 3 is a control to verify the presence of maternal lethal activity and zygotic rescue activity on a nonirradiated $M^{1}$ chromosome. Testcross 4 is to confirm that the $M^{I R}$ au revertant chromosome has lost maternal lethal activity. 
most prevalent. Of the 113 strains, $42(=37 \%)$ representing 14 countries contained $M^{4}$, whereas $11(=10 \%)$ representing nine countries had $M^{1}$ and $13(=12 \%)$ representing six countries appeared to carry other Medea factors, based on the presence of maternal lethal activity that was not rescuable by either $M^{1}$ or $M^{4}$. Most strains tested from Africa, South and Central America and south-east Asia contained these new Medea-like factors, whereas they were absent from North America, Europe, Australia and the Indian subcontinent. However, they appeared to have variable penetrance, and their existence could not be consistently confirmed in subsequent tests. Thus, they are not included in Table 5 . Major geographical differences in Medea distribution are evident from the data in Table 5. North America and Europe have a high incidence of $M^{4}$ and a low incidence of $M^{1}$; Australia and the Indian subcontinent have a low incidence of Medea factors of either type; whereas South America, Africa and south-east Asia have a high incidence of both types. If the 55 strains from Australia and the Indian subcontinent are excluded, $M^{4}$ occurs in 38 of 58 , or $66 \%$ of all remaining strains. $M^{1}$ occurred in 11 of $28(=39 \%)$ of all strains tested from South America, Africa and south-east Asia, but in none of 52 strains tested from North America, Europe (including the Middle East), Australia and the Indian subcontinent. In North America we observed a distinct regional nonuniformity in $M^{4}$ distribution (data not shown). $M^{4}$ factors were found in all 11 strains originating from the midwest or northern plains, but in none of the five strains originating from the deep south.

\section{$M^{1}$ always co-occurs with $M^{4}$}

It is notable that $M^{4}$ was present in all $11 M^{1}$ strains, whereas only $30 \%$ of the non- $M^{1}$ strains carried $M^{4}$.

Table 5 World distribution of Medea types

\begin{tabular}{lcrrr}
\hline & \multicolumn{4}{c}{$\begin{array}{c}\text { Number of strains with indicated } \\
\text { Medea } \text { genes }\end{array}$} \\
\cline { 2 - 5 } Continent $\dagger$ & $M^{l}+M^{4}$ & $M^{4}$ & None & Total \\
\hline NA & 0 & 12 & 6 & 18 \\
EU & 0 & 8 & 4 & 12 \\
AU & 0 & 4 & 18 & 22 \\
IN & 0 & 0 & 33 & 33 \\
SA & 4 & 3 & 1 & 8 \\
AF & 2 & 1 & 2 & 5 \\
AS & 5 & 3 & 7 & 15 \\
Totals & 11 & 31 & 71 & 113 \\
\hline
\end{tabular}

$\dagger$ Abbreviations NA, North America; EU, Europe; AU, Australia; IN, Indian subcontinent; SA, South and Central America; AF, Africa; AS, south-east Asia.
Although $M^{1}$ was never detected in the absence of $M^{4}$ in nature, the two could be readily separated by genetic segregation in the laboratory. Purified $M^{1}$ strains appear to be fully viable and the isolated $M^{l}$ factor is stable and functional.

\section{Discussion}

Although we first reported the existence of Medea factors as long ago as 1992, the detailed workings of this unprecedented mechanism for self-propagation of parasitic DNA remain obscure. The present work poses or leaves unanswered a number of intriguing questions. What could explain the patchy distribution of Medea elements in nature? Why is $M^{1}$ never found in the absence of $M^{4}$ ? Are $M$ factors of recent evolutionary origin? Are they foreign elements or endogenous genes? Are they dispensable? Are they unique to the genus Tribolium?

We hoped that the question of whether $M$ genes are dispensable would be answered by reversion analysis. If independently derived revertants of a particular $M$ gene are lethally noncomplementary, then that gene is probably vital. As we obtained only one revertant, this question must be revisited in the future with additional reversion analysis.

The fact that the $M^{1}$ revertant chromosome had lost maternal lethal activity while retaining zygotic rescue activity confirms the bifunctional nature of this locus. A Medea locus could be two separate but closely linked genes (encoding a maternal poison and a zygotic antidote, respectively) that selection favours to be maintained in linkage disequilibrium. Such a gene pair might best thrive in a recombination-suppressed region, for example, near centromeric heterochromatin. Although cytological positioning of Medea loci has not been accomplished, $M^{1}$ and the closely linked $M^{3}$ have been recombinationally mapped to one extreme end of metacentric LG3, and thus are probably much nearer a telomere than a centromere.

Until recently all evidence suggested that each $M$ locus is fully independent of all other $M$ loci, i.e. each $M$ factor expresses maternal lethal and zygotic rescue activity independently of other $M$ factors, and rescue activity of a given $M$ factor does not protect against the maternal lethal activity of a different $M$ factor. In 1996, however, we discovered that the hybrid incompatibility factor $H$ (Thomson et al., 1995) interacts lethally with both $M^{1}$ and $M^{4}$. That is, $H /+$ heterozygotes die prior to adult development if they also carry a copy of either $M^{1}$ or $M^{4}$ (Thomson \& Beeman, 1997). More detailed examination of this phenomenon could give new insight into the Medea mechanism. 
In geographical regions where Medea is at or near fixation, such as $M^{4}$ in the midwestern United States, its selfishness is silenced and the element remains hidden. Similarly, it has been reported that sex-ratio distorter is widespread but phenotypically silenced in natural populations of Drosophila simulans (Atlan et al., 1997). These observations lend support to the idea that genetic conflicts in general might be more common than often considered (Hurst \& Hurst, 1996). It would be remarkable if maternal-effect selfish genes, represented by at least four different loci in the only species to be carefully scrutinized, were not much more widespread than is now apparent.

A full understanding of the Medea system will not be achieved without cloning and sequencing at least one $M$ locus. Because the Medea mechanism appears to be unprecedented, as no homologues are known in other species, and as we have no DNA sequence information, it would seem that positional cloning might be the easiest approach. The feasibility of such a strategy is now under investigation.

\section{References}

ATLAN, A., MURCOT, H., LANDRE, C. AND MONTCHAMP-MOREAU, C. 1997. The sex-ratio trait in Drosophila simulans: Geographical distribution of distortion and resistance. Evolution, 51, 1886-1895.

BeEMAN, R. w. 1986. Section on new mutants. Tribolium Inf. Bull., 26, 83 .
BeEMAN, R. W. AND StUART, J. J. 1990. A gene for lindane + cyclodiene resistance in the red flour beetle (Coleoptera: Tenebrionidae). J. Econ. Entomol., 83, 1745-1751.

BEEMAN, R. W., FRIESEN, K. S. AND DENELL, R. E. 1992. Maternaleffect, selfish genes in flour beetles. Science, 256, 89-92.

HALISCAK, J. P. AND BEEMAN, R. W. 1983. Status of malathion resistance in five genera of beetles infesting farm-stored corn, wheat and oats in the United States. J. Econ. Entomol., 76, 717-722.

HURST, L.D. 1993. scat $^{+}$is a selfish gene analogous to Medea of Tribolium castaneum. Cell, 75, 407-408.

HURST, L. D. AND HURST, G. D. D. 1996. Evolutionary genetics: genomic revolutionaries rise up. Nature, 384, 317-318.

MOCELIN, G. AND STUART, J. J. 1996. Crossover suppressors in Tribolium castaneum. J. Hered., 87, 27-34.

PETERS, L. L. AND BARKER, J. E. 1993. Novel inheritance of the murine Severe Combined Anemia and Thrombocytopenia (scat) phenotype. Cell, 74, 135-142.

SOKOLOFF, A. 1962. Linkage studies in Tribolium castaneum Herbst. V. The genetics of Bar eye, microcephalic and Microphthalmic, and their relationships to black, jet, pearl and sooty. Can. J. Genet. Cytol., 4, 409-425.

THOMSON, M. S. AND BEEMAN, R. W. 1999. Assisted suicide of a selfish gene. J. Hered., 90, 191-194.

THOMSON, M. S., FRIESEN, K. S., DENELL, R. E. AND BEEMAN, R. W. 1995. A hybrid incompatibility factor in Tribolium castaneum. J. Hered., 86, 6-11.

WADE, M. J. AND BEEMAN, R. W. 1994. The population dynamics of maternal-effect selfish genes. Genetics, 138, 1309-1314.

WEICHENHAN, D., TRAUT, W., KUNZE, B. AND WINKING, H. 1996. Distortion of Mendelian recovery ratio for a mouse HSR is caused by maternal and zygotic effects. Genet. Res., 68, 125129. 